\title{
P127 Graded Cuff Compression Protocol to Induce Retrograde Shear Produces Proportionate Increment in Regional Arterial Compliance in Human Peripheral Artery
}

\author{
Naaz Afreen ${ }^{1, *}$, Dinu Chandran ${ }^{1}$, Ashok Jaryal ${ }^{1}$, Kishore Deepak $^{1}$, Sitikantha Roy ${ }^{2}$ \\ ${ }^{1}$ All India Institute of Medical Sciences, New Delhi, India \\ ${ }^{2}$ Indian Institute of Technology, New Delhi, India
}

\section{ABSTRACT}

Background: External compression using pneumatic cuff inflation is a well-established model used to induce incremental changes in proximal retrograde flow and shear [1]. The mechanisms behind distal cuff compression leading to proximal increase in retrograde shear are not clear. We hypothesized that changes in regional arterial compliance due to external cuff compression lead to changes in pulse waveform and pulse wave reflections proximal to the cuff compression.

Methods: We calculated brachial-radial pulse wave velocity (br-PWV) as a measure of regional arterial compliance in the area undergoing external cuff compression. This was accomplished using simultaneous two-point applanation tonometry over the cubital fossa and the wrist (Figure 1), while pneumatic cuff compression at 25, 50, and $75 \mathrm{mmHg}$ (3-minute compression, randomized order, separated by 5 minutes) was applied around the middle of the forearm in 9 healthy young subjects (27 \pm 4 years). Br-PWV was calculated using foot detection of the pulse waveforms and brachial-radial distance.

Results: External cuff compression at 50 and $75 \mathrm{mmHg}$ resulted in significant fall in br-PWV but not at $25 \mathrm{mmHg}$. Percentage change in br-PWV showed graded decrement with rise in pressure of external cuff compression $(-1.16 \pm 6.96$ at $25 \mathrm{mmHg}$, $-10.57 \pm 3.80$ at $50 \mathrm{mmHg}$ and $-35.59 \pm 11.08$; all comparisons $p<0.05)$.

Conclusion: External cuff compression leads to changes in regional arterial compliance, which possibly affect the pressure wave reflections and pressure waveform proximally, leading to changes in the blood flow pattern at the site.

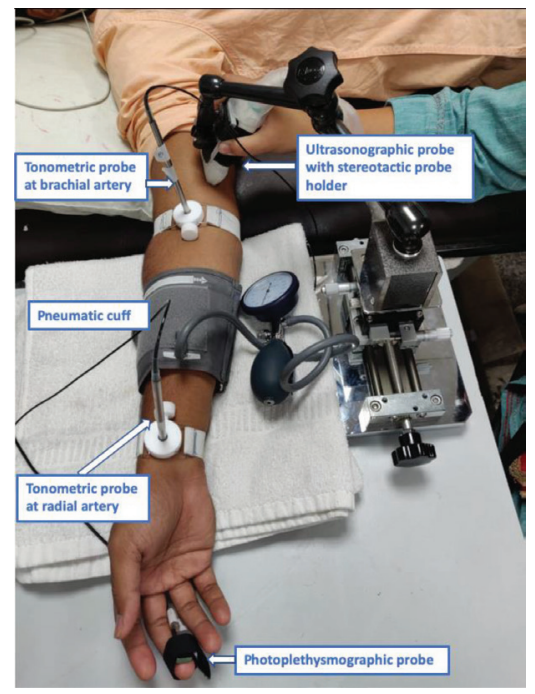

\section{REFERENCE}

Figure 1

[1] Thijssen DH, Dawson EA, Tinken TM, Cable NT, Green DJ. Retrograde flow and shear rate acutely impair endothelial function in humans. Hypertension 2009;53:986-92.

(c) 2019 Association for Research into Arterial Structure and Physiology. Publishing services by Atlantis Press International B.V. This is an open access article distributed under the CC BY-NC 4.0 license (http://creativecommons.org/licenses/by-nc/4.0/). 